\title{
Research on the Model and Application of Virus Contact and Transmission in Cabin
}

\author{
Yanxi Liu ${ }^{1, ~ a ~, ~ L i ~ W a n g ~}{ }^{2, b}$, Liangwen Zheng ${ }^{3, c}$ and Qing Liu ${ }^{1, d}$ \\ ${ }^{1}$ Civil Aviation University of China, Tianjin 300300, China; \\ ${ }^{2}$ Shanghai International Airport Services, Shanghai 200000, China; \\ ${ }^{3}$ Hunan Airport Corporation, Changsha 410000, China. \\ a260291036@qq.com, b17612236496@163.com, crzlw971025@163.com, ${ }^{\mathrm{d}}$ 171706882@qq.com
}

Keywords: Virus spread, cabin, contact spread, MATLAB simulation.

\begin{abstract}
A cellular automata model is established for the virus in the cabin through contact and transmission. The probability of virus transmission and infection in the cabin is analyzed. The curve of the virus change in the cabin is obtained by using MATLAB to solve the problem. Then, effective intervention measures are proposed to reduce The probability of the virus spreading through contact in the cabin.
\end{abstract}

\section{Background}

Air travel brings people from different geographical environments together. Due to the different geographical environments in different regions, people's immunity and acceptability are different. The virus in the cabin is mainly transmitted through contact, air, and media. For passengers and crew, contact and transmission are divided into direct contact and indirect contact. Passengers will carry the virus to different places, which will lead to the rapid spread of the virus and cause economic losses to people.

\section{Cogitation of the Research}

The SIR cellular automata model of virus contact and transmission was established. The MATLAB program was used to obtain the change curve of infected persons, susceptible persons, and immune persons after the virus was transmitted through contact in a unit time. Passengers are divided into susceptible persons, infected persons and immunized persons. The state of the cell, the state of the cell neighbor and the evolution rules are determined, and the actual situation is realized through computer simulation. By comparing the results obtained by the two models, analyzing the differences in the data obtained by the two methods, determining an optimal method, and then proposing some improved measures to remind passengers what behaviors may cause them to be infected by the virus in order to reduce Probability of virus transmission.

\section{Research Program}

\subsection{Construction of SIR Cellular Automata Model}

The SIR model is to classify the population infected by the virus in a certain space. The number of susceptible, infected, and immune populations at time $\mathrm{t}$ are $S(\mathrm{t}) 、 I(\mathrm{t}) 、 R(\mathrm{t})$, and are defined by cellular automata and are represented by mathematical symbols $C=\left(L_{\propto}, S, N, f\right)$, Among them, the $L_{\alpha}$ means cell is all the people, $S$ is the state set, $N$ is the neighbor of the cell, and $\mathrm{f}$ is the evolution rule of the cell. The established cellular automaton model is as follows.

(1) Cell space: two-dimensional cell space. 
(2) Neighbor form: Moore type with a neighbour radius of 1 , and the eight surrounding neighbours are expressed in order as:

$$
\begin{array}{ccc}
(i-1, j-1) & (i-1, j) & (i-1, j+1) \\
(i, j-1) & (i, j) & (i, j+1) \\
(i+1, j-1) & (i+1, j) & (i+1, j+1)
\end{array}
$$

(3)Cell evolution rules: The initial state of all cells is $S=0$, and the state of the pathogen is set to 1. The sick infected the susceptible at the rate of infection, and exchanged randomly at a probability of. Scan all cells from time 0, and update the cell status according to the following rules.

When $S_{i, j}^{t}=0$ (susceptible),

the cell at (i,j),its Adjacency matrix

$$
M=\left\{\begin{array}{ccc}
S_{i-1, j-1}^{t} & S_{i-1, j}^{t} & S_{i-1, j+1}^{t} \\
S_{i, j-1}^{t} & S_{i, j}^{t} & S_{i, j+1}^{t} \\
S_{i+1, j-1}^{t} & S_{i+1, j}^{t} & S_{i+1, j+1}^{t}
\end{array}\right\}
$$

matrix $N=\left\{\begin{array}{ccc}C_{i-1, j-1}^{t} & C_{i-1, j}^{t} & C_{i-1, j+1}^{t} \\ C_{i, j-1}^{t} & C_{i, j}^{t} & C_{i, j+1}^{t} \\ C_{i+1, j-1}^{t} & C_{i+1, j}^{t} & C_{i+1, j+1}^{t}\end{array}\right\}$ is the cell of S=1(Sick) in the matrix M.

Calculate the probability of cell infection at (i, j):

$$
P=\frac{k \times\left(C_{i-1, j}^{t}+C_{i+1, j}^{t}+C_{i, j-1}^{t}+C_{i, j+1}^{t}\right)}{4}+\frac{1 \times\left(C_{i-1, j-1}^{t}+C_{i+1, j-1}^{t}+C_{i-1, j+1}^{t}+C_{i+1, j+1}^{t}\right)}{4}
$$

Where $\mathrm{k}$ is the influence value of upper, lower, left, and right on the cell at (i, j), l is the influence value of upper left, lower left, upper right, and lower right on the cell at (i, j), $k>1$. Therefore, the cells at (i,j) change from state $S_{\mathrm{i}, j}^{t}=0$ (susceptible) to $S_{\mathrm{i}, j}^{t}=1$ (disease) with probability P, and the individual's onset time is $t_{n}\left(S_{\mathrm{i}, \mathrm{j}}^{t}\right)=\mathrm{t}_{n}\left(S_{i, j}^{t}\right)+1$.

When $S_{i, j}^{t}=1$ (infected),

Judgment $t_{n}\left(S_{i, j}^{t}\right)$ values are needed to determine whether passengers are susceptible, infected, or immune. At that time, the cells at (i, j) were in the $S_{i, j}^{t}=2$ (immune) state. At this time, the maximum value of the passenger's onset time is $t_{n}\left(S_{\mathrm{i}, \mathrm{j}}^{t}\right)=0$, the maximum value of the passenger's immune time is $t_{\mathrm{m}}\left(S_{\mathrm{i}, \mathrm{j}}^{t}\right)=\mathrm{t}_{m}\left(S_{i, j}^{t}\right)+1$; otherwise, the cells at (i, j) were $S_{\mathrm{i}, j}^{t}=1$ (the disease ) State, the maximum time of passenger onset is $t_{n}\left(S_{i, j}^{t}\right)=\mathrm{t}_{n}\left(S_{i, j}^{t}\right)+1$.

When $S_{i, j}^{t}=2$ (immune),

Judgment $t_{\mathrm{m}}\left(S_{\mathrm{i}, \mathrm{j}}^{t}\right)$ values are needed to determine whether passengers are susceptible, infected, or immune. When $\left.t_{\mathrm{m}}\left(S_{\mathrm{i}, \mathrm{j}}^{t}\right)\right\rangle t_{m}$, the cells at (i,j) were in $S_{\mathrm{i}, j}^{t}=0$ (susceptible) state, and the maximum value of passenger immune time is $t_{\mathrm{m}}\left(S_{\mathrm{i}, \mathrm{j}}^{t}\right)=0$; otherwise, the cells at (i, j) were in $S_{\mathrm{i}, j}^{t}=2$ (immunity) state, at which time the passenger was immune. The maximum value is $t_{m}\left(S_{i, j}^{t}\right)=\mathrm{t}_{m}\left(S_{i, j}^{t}\right)+1$.

(4) The above rules are established with passengers fixed in their seats. The following is a simple analysis of passengers in the aisle. For the operation of passengers walking randomly, we can assume that the maximum step size of passengers walking randomly is Q, fix this step size, set a random scan order for all cells, and then randomly scan all cells in this order. For the randomly scanned cell, two random numbers $d_{i}$ and $d_{j}$ are generated, and the cell $C_{i, j}$ at (i, j) is exchanged with the cell $C_{i+d_{i}, j+d_{j}}$ at $\left(i+d_{i}, j+d_{j}\right)$ to complete a move. 


\subsection{Application of SIR Cellular Automata Model}

Assume that the number of cells in the model is 167, time is 60 unit times, the infection rate $P_{1}$ is 0.075, and the probability of random exchange $P_{2}$ is 0.001 . Based on the internal seat map of the selected model B737-800, we randomly assumed that one of the passengers was an infected person, as shown in Figure 1, and then studied the infection of eight passengers around it.

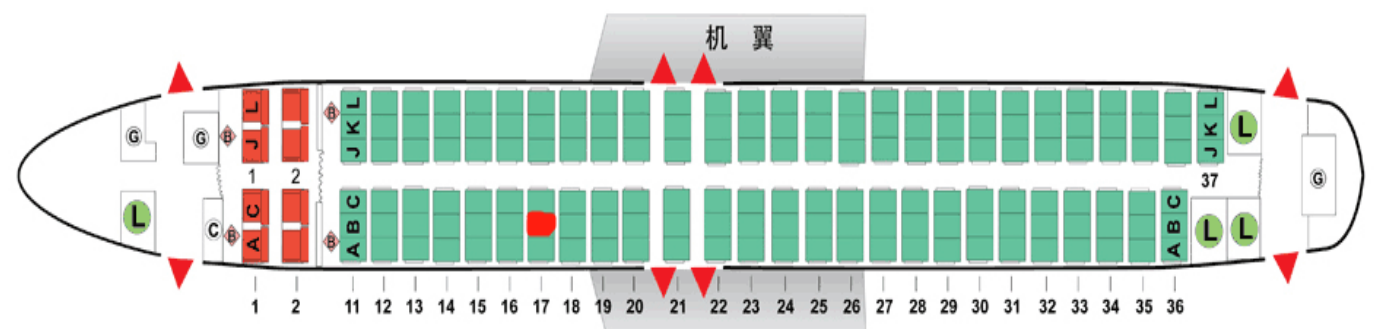

Fig. 1 Seat of a randomly infected person in a cellular automaton

Using MATLAB to solve the cellular automata, the SIR cellular automata model is solved. The time-varying curve of the model obtained with MATLAB software is shown in Figure 2.

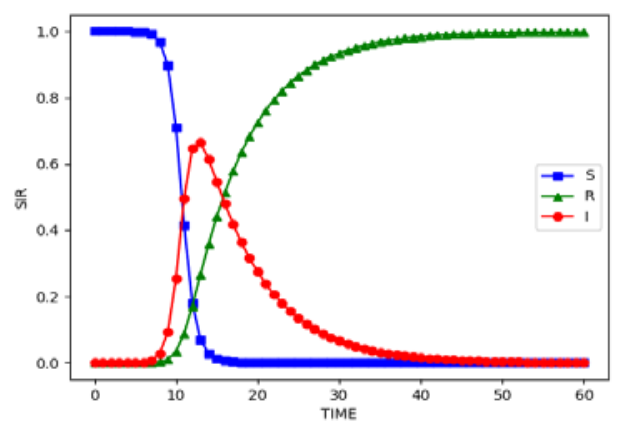

Fig. 2 Curve change of SIR cellular automata model

The values of some infected persons, susceptible persons, and immunized persons over time were selected over 60 unit times, as shown in Table 1.

Table 1 The probability of infection, susceptibility, and immunization over time

\begin{tabular}{cccccccccc}
\hline Unit of time & 0.00 & 5.00 & 10.00 & 20.00 & 30.00 & 40.00 & 50.00 & 55.00 & 60.00 \\
\hline Infected & 0.01 & 0.01 & 0.09 & 0.316 & 0.076 & 0.018 & 0.004 & 0.002 & 0.000 \\
Susceptible & 0.99 & 0.99 & 0.97 & 0.018 & 0.001 & 0.001 & 0.001 & 0.001 & 0.001 \\
Immunizer & 0.00 & 0.00 & 0.01 & 0.683 & 0.924 & 0.982 & 0.996 & 0.998 & 0.999 \\
\hline
\end{tabular}

\section{Results}

Based on the assumptions of the model parameters, the change curve of the infected person in the unit time of SIR obtained by running with MATLAB. During the process of virus contact and transmission in the cabin, the following conclusions can be obtained by using the SIR cellular automaton model:

(1) In the simulated 60 unit time, it can be seen from the image that the number of infected passengers in the cabin first increases and then decreases to reach a stable state; the susceptible person gradually decreases from the maximum value and then reaches a stable state; The immunized person gradually increases from zero to the maximum, and then maintains a steady state.

(2) The image shows that the maximum value of the infection is around 9unit times. It can be used during the time period during which passengers are boarding, placing luggage and seating, so 
there is a lot of contact with passengers during this time. Opportunities, including nearby passengers, passing passengers, and flight attendants.

(3) After the 10th unit time, the passenger sat firmly and relatively reduced the chance of contact. At this time, only the cabin crew and the passengers going to the toilet will contact each other, resulting in the transmission of the virus. So after that the curve shows a downward trend to the final plateau.

According to the above conclusions, compared with the actual situation, the number of early infections gradually increased, then decreased, and finally stabilized. These are basically consistent, so in the cabin, the time for the virus to spread through contact is mostly when the passengers board the plane. After the plane is stable.

\section{Summary}

The virus spreads quickly in the cabin through contact. If the possibility of such transmission cannot be reduced, passengers will carry the virus to every area after disembarking, causing quite serious consequences. Therefore, comprehensive consideration should be taken to prevent the possibility of virus transmission in the cabin from contact with precautions before boarding, on-board precautions, aircraft cleaning and disinfection, and aircraft ventilation.

\section{Acknowledgements}

Nature Fund name: Simulation of typical virus propagation in civil aircraft cabins and emergency strategy research project approval number: U1633123 Application code: F01

\section{References}

[1] H.Lei, Y.Li, et al. Routes of transmission of influenza A H1N1, SARS CoV, and norovirus in air cabin: Comparative analyses. Department of Mechanical Engineering: 2017.

[2] You Aili. SIR epidemic model based on cellular automata [D]. (Fine paper). Xinjiang: Xinjiang University, 2010.

[3] Zheng Sanqiang, Han Xiaozhuo. Research on Cellular Automata Simulation of SIR Epidemic Model under Multi-Factor Relatives [J]. Journal of Guangdong University of Technology: Engineering Science Edition, 2018, 35 (5).

[4] Li Gang, Yu Lei and others. Research on the spread model of infectious diseases based on cellular automata [J]. Computer Engineering and Application, 2007, 43 (2).

[5] Luo Jia, Bai Chunxue. The effect of air travel on respiratory physiology and common diseases [J]. Chinese Journal of Internal Medicine, 2006, 45 (12). 